\title{
Vascular endothelial ERp72 is involved in the inflammatory response in a rat model of skeletal muscle injury
}

\author{
NOUREDDINE BEN KHALAF, DALAL AL-MEHATAB and DAHMANI M. FATHALLAH \\ Department of Life Sciences, Health Biotechnology Program, College of Graduate Studies, \\ King Fahd Chair for Health Biotechnology, Arabian Gulf University, Manama 329, Bahrain
}

Received July 19, 2020; Accepted November 25, 2020

DOI: $10.3892 / \mathrm{mmr} .2021 .11825$

\begin{abstract}
The vascular inflammatory response involves the coordinated action of a large network of molecular mediators and culminates in the transmigration of leukocytes into the site of inflammation. Inflammatory mediators include a variety of protein families, including adhesion molecules such as integrins and members of the immunoglobulin superfamily, as well as other cytokines and chemokines. In this study, a rat model of traumatic skeletal muscle injury was used to demonstrate endoplasmic reticulum resident protein 72 (ERp72) overexpression in the early phase of the inflammatory response that follows skeletal muscle injury. Reverse transcription-quantitative PCR, western blotting, dual-labeling immunohistochemistry and immunofluorescence experiments confirmed that ERp72 was expressed on the endothelial cells of blood vessels present at the injured area. In addition, a cell-based neutrophil adhesion assay indicated that a polyclonal antibody specific for ERp72 significantly reduced adhesion of neutrophils to activated human umbilical vein endothelial cells (35\% reduction). These data suggested that ERp72 expression on vascular endothelial cells may play a role in skeletal muscle inflammation and could be considered as a target for the modulation of leukocyte-endothelial cell interactions in an inflammatory setting.
\end{abstract}

Correspondence to: Professor Dahmani M. Fathallah, Department of Life Sciences, Health Biotechnology Program, College of Graduate Studies, King Fahd Chair for Health Biotechnology, Arabian Gulf University, Building 293, Road 2904, Manama 329, Bahrain

E-mail: d.fathallah@agu.edu.bh

Abbreviations: BCECF-AM, 2'7'-bis-(2-carboxyethyl)-5(and-6)carboxyfluorescein acetoxymethyl; ER, endoplasmic reticulum; HBSS, Hank's balanced salt solution; HUVEC, human umbilical vein endothelial cell; NPH, neutrophil; PDI, protein disulfide isomerase; PMN, polymorphonuclear neutrophil

Key words: endothelial cells, neutrophil, inflammation, adhesion molecules

\section{Introduction}

The inflammatory response that follows tissue injury mobilizes a large network of molecular mediators. The coordinated and finely tuned actions of these mediators culminate in the recruitment of leukocytes to the site of injury; a cascade of events regulates leukocyte recruitment and trafficking to the site of injury. Adhesion molecules, such as selectins and integrins, control the process of initial rolling, firm adhesion, crawling and transmigration $(1,2)$. Intracellular adhesion molecule-1 (ICAM-1) expressed on the surface of endothelial cells interacts with activated $\alpha \mathrm{M} \beta 2$ and $\alpha \mathrm{L} \beta 2$ integrins and allows neutrophils to crawl and firmly adhere to activated endothelium (3). Furthermore, disulfide bonds are known to be a key regulator of protein flexibility and function (4), and it has been reported that disulfide bond reduction $(5,6)$ or cysteine mutation $(7,8)$ modulates integrin activation and neutrophil adhesion. A previous study by Hahm et al (9) demonstrated that extracellular protein disulfide isomerase (PDI) regulated the ligand-binding activity of $\alpha \mathrm{M} \beta 2$ integrin and neutrophil recruitment during vascular inflammation through its isomerase activity. Another recent study by Rosenberg et al (10) using three different cell lines that depend on adhesion for survival indicated that PDI was involved in integrin-mediated adhesion, through the catalysis of disulfide bond exchange and enhancement of cell adhesion by both its oxidoreductase and chaperone activities (10). These data suggest that extracellular PDI may be a novel target for the modulation of leukocyte trafficking.

PDI, also known as the $\beta$ subunit of prolyl 4-hydroxylase, is a $55-\mathrm{kDa}$ soluble protein that constitutes the archetype of the PDI family of proteins, which contains a thioredoxin-like $\beta \alpha \beta \alpha \beta \alpha \beta \beta \alpha$ fold motif and acts as a dithiol-disulfide oxidoreductase to catalyze the reduction, oxidization and isomerization of disulfide bonds (11). PDI also acts as a molecular chaperone both in vitro (12) and in vivo (13). The PDI family comprises $>20$ members that vary in length and structural arrangement, with most PDI members sharing catalytic and non-catalytic thioredoxin-like domains (14). All members are localized in the ER where they contribute to ER homeostasis by maintaining an oxidative environment (15). PDI is organized into four thioredoxin-like domains, a, a', b and b', in addition to a linker domain, $x$. The catalytic domains a and a' contain Cys-Gly-His-Cys motifs 
that react with thiol groups in substrate proteins, whereas $\mathrm{b}$ and $\mathrm{b}^{\prime}$ are considered as non-catalytic domains and are involved in substrate recognition and recruitment (14). The most commonly studied members of the PDI family after PDI are endoplasmic reticulum resident protein (ERp)57, ERp72, ERp29, ERp44, and PDIA2 (11). The difference between the PDI family members is protein length and structural arrangement of active and inactive domains (14).

Although the chaperone function of PDIs is generally mapped to the endoplasmic reticulum (ER) (16), one previous study demonstrated extracellular localization and function of PDI on the surface of several cell types, suggesting an enzymatic mediation for disulfide exchange in the cell-surface receptors (17). Indeed, PDIs expressed on the surface of leukocytes and platelets are involved in hemostasis, vascular inflammation and thrombosis (18-20). Moreover, PDI, ERp5 and ERp57 are involved in the initiation of thrombus formation following laser-induced vascular injury in vivo (21-23), in which endothelial cells and platelets are activated and secrete PDI and other thiol isomerases (24). ERp72 was shown to initiate coagulation and promote thrombosis formation through a cascade of reactions in platelets (25), which promotes tumor progression (26-28). In addition, ERp72 knockdown impaired platelet function and fibrin formation in mice (29). PDI is also associated with thrombus growth through the regulation of $\beta 3$ integrins $(17,18,30,31)$. Inhibition of PDI with a blocking antibody completely inhibits both platelet thrombus formation and fibrin generation $(17,22,32)$.

To the best of the authors' knowledge, the majority of studies are focused on PDIs in leukocytes and little to almost no information is available on the role played by endothelial PDIs during cellular activation and recruitment. The present study was initiated following a yeast two-hybrid screening of the binding partner of a biological compound (SI/0220) that is currently under development (Patent FR2909672A1, pending). SI/0220 is a bispecific integrin/selectin biological compound that was engineered to target ICAM1 and P-selectin glycoprotein ligand-1 $(1,2)$. These two endothelial cell surface receptors are the natural ligands of the leukocyte adhesion molecules CD11b/CD18 (33) and L-selectin (34), respectively, which are the main contributors to leukocyte extravasation in inflamed tissues. Unpublished data have suggested that SI/0220 has anti-inflammatory activity and modulates polymorphonuclear neutrophil transmigration. The screening against a library of TNF $\alpha$-activated human umbilical vein endothelial cells (HUVECs) identified ERp72 as a major binding partner of SI/0220. To validate this observation, ERp72 gene expression was assessed in vascular endothelial cells in a rat model of inflammatory skeletal muscle injury (35). ERp72 was overexpressed from the early phase following injury. For validation, the present study used western blotting, immunohistochemistry and immunofluorescence experiments in the endothelium of blood vessels post-injury, in addition to adhesion inhibition assays of neutrophils to TNF $\alpha$-activated HUVECs. These findings suggested that endothelial ERp72 may mediate the inflammatory response through the regulation of neutrophil adhesion during their recruitment to the inflammation site and could constitute a novel target to modulate the overflux of activated leukocytes to the site of injury.

\section{Materials and methods}

Yeast two-hybrid analysis. Yeast two-hybrid screening was performed using the Hybrigenics Services technical platform (http://www.hybrigenics-services.com). The technology is based on the reconstitution of a functional transcription factor followed by the expression of a reporter gene in genetically modified yeast cells. The coding sequence for SI/0220 (data not shown; patent pending) was amplified by PCR and cloned into pB27 plasmids as a C-terminal fusion to LexA (N-LexA-SI/0220-C). The construct was checked by sequencing the entire insert and used as a bait to screen a randomly-primed HUVEC cDNA library constructed into pP6 and pB27 plasmids derived from the original pBTM116 (36) and pGADGH (37) plasmids, respectively. A total of 113 million clones (10-fold the complexity of the library) were screened using a mating approach with YHGX13 (Y187 ade2-101:loxP-kanMX-loxP, MAT $\alpha$ ) and L40 $\Delta$ Gal4 (MATa) yeast strains as previously described (38). Cells were incubated in rich medium for $4.5 \mathrm{~h}$ at $30^{\circ} \mathrm{C}$ and $\mathrm{His}^{+}$colonies were selected on a plates made from medium lacking tryptophan, leucine and histidine. The prey fragments of the positive clones were amplified by PCR and sequenced at their 5' and 3 ' junctions. The resulting sequences were used to identify the corresponding interacting proteins in the GenBank database (National Center for Biotechnology Information) using a fully automated procedure. A confidence score (Predicted Biological Score; PBS) was attributed to each interaction as previously described (39). For protein annotation, conserved domains were predicted using the Pfam v.32.0 (https://pfam.xfam.org) and SMART v.8 (http://smart.embl-heidelberg.de) servers. The transmembrane domain was predicted using the TMHMM server v.2.0 (http://www.cbs.dtu.dk/services/TMHMM). The signal peptide was predicted using the SignalP v. 3.0 server (http://www.cbs.dtu.dk/services/SignalP-3.0). The coiled coil domain was predicted using the COILS v.2.2 server (https://embnet.vital-it.ch/software/COILS_form.html).

Primer design. Rattus norvegicus mRNA sequences for ERP72 (PDIA4), ICAM1, VCAM1, and SELE were downloaded from the Uniprot server (release 2019_11, https://www.uniprot.org). Specific primers were designed using the Primer3 web server v.0.4.0 (http://bioinfo.ut.ee/primer3-0.4.0) with default parameters, and their specificity was assessed using BLAST v.2.9.0 (https://blast.ncbi.nlm.nih.gov) for cross-priming. Primers used for reverse-transcription-quantitative (RT-q) PCR (Table I) were purchased from Biolegio B.V. in lyophilized form. All primers were synthesized at a $40 \mathrm{nmol}$ scale and purified by high-performance liquid chromatography. The primers were resuspended in nuclease-free water at a concentration of $100 \mu \mathrm{M}$.

Animals. A total of 30 female inbred Wistar rats weighing 200-220 g were used to develop the muscle injury model, following institutional guidelines and in conformity with the international standards recommended for animal experimentation. All animal experimental protocols were approved by the Research and Ethics Committee of Arabian Gulf University (Manama, Bahrain). All methods were carried out in accordance with the committee's relevant guidelines and regulations 
Table I. List of primers used for reverse transcription-quantitative PCR.

\begin{tabular}{|c|c|c|c|c|}
\hline Primer name & Target gene & Primer sequence $\left(5^{\prime} \rightarrow 3^{\prime}\right)$ & Melting temperature, ${ }^{\circ} \mathrm{C}$ & Product size, bp \\
\hline PDI4F1 & ERP72 & TGCAGCCTGAGAAGTTCCAG & 59.96 & 200 \\
\hline PDI4R1 & ERP72 & GCTGAAGTCCACGCTGTAGT & 60.04 & 200 \\
\hline ICAMF & ICAMI & GGTATCCATCCATCCCACAG & 60.01 & 208 \\
\hline ICAMR & ICAMI & GCCACAGTTCTCAAAGCACA & 60.03 & 208 \\
\hline VCAMF & VCAM1 & ACAAAACGCTCGCTCAGATT & 60.02 & 152 \\
\hline VCAMR & VCAM1 & GTCCATGGTCAGAACGGACT & 59.97 & 152 \\
\hline E-seleF & SELE & TTTTTGGCACGGTATGTGAA & 59.97 & 168 \\
\hline E-seleR & SELE & AGGTTGCTGCCACAGAGAGT & 60.06 & 168 \\
\hline GAPDHF & $G A P D H$ & CTCATGACCACAGTCCATGC & 59.80 & 155 \\
\hline GAPDHR & $G A P D H$ & TTCAGCTCTGGGATGACCTT & 59.90 & 155 \\
\hline
\end{tabular}

ERP72, endoplasmic reticulum resident protein 72; F, forward; ICAM1, intercellular adhesion molecule 1; R, reverse; SELE, E-selectin; $V C A M 1$, vascular cell adhesion molecule 1 .

in March 2019. A total of 25 rats were used for the monitoring of gene expression and immunohistochemistry experiments post-injury. The remaining five rats did not undergo muscle injury and were used as an untreated control group.

Rat skeletal muscle injury model. Rat skeletal muscle injury was performed as described previously (35). Animals were anesthetized intraperitoneally using a mixture of $90 \mathrm{mg} / \mathrm{kg}$ ketamine and $10 \mathrm{mg} / \mathrm{kg}$ xylazine. Anesthesia induction was confirmed by lack of pedal reflex. The muscles in both limbs were punctured using a 20-gauge needle mounted on a manual leather-puncturing device to create a hematoma as previously described (35). The rats were euthanized using $\mathrm{CO}_{2}$ at a displacement at a flow rate of $50 \%$ at different timepoints varying from $15 \mathrm{~min}$ to $4 \mathrm{~h}$ post-injury. Animal death was verified by ascertaining cardiac and respiratory arrest. A surgical procedure was then used to extract blood vessels in the injured area from all rats, vessels were further dissected under a microscope to remove all irrelevant tissues. Blood vessels were then stored in TRIzol ${ }^{\circledR}$ (Invitrogen; Thermo Fisher Scientific, Inc.) for RNA and protein extraction. For histology, wounded muscles were resected, fixed in $10 \%$ formalin overnight at $4^{\circ} \mathrm{C}$ and paraffin-embedded, then cut to $4-5 \mu \mathrm{m}$ sections. The sections were used for immunohistochemistry and stained at room temperature for $3 \mathrm{~min}$ and $45 \mathrm{sec}$ with hematoxylin and eosin, respectively, for light microscopy examination. Histological observations were performed on a Zeiss Axioskop light microscope (Carl Zeiss AG) using an eyepiece graticule grid.

Gene expression analysis. Total mRNA was extracted from homogenized tissues using the TRIzol ${ }^{\circledR}$ extraction protocol (Invitrogen; Thermo Fisher Scientific, Inc.) and reverse transcribed using the ProtoScript ${ }^{\circledR}$ First Strand cDNA Synthesis kit (New England BioLabs, Inc.) according to the manufacturer's instructions. Primer sets for RT-qPCR (Table I) were used to amplify target regions from cDNA as templates using the GoTaq DNA polymerase (Promega Corporation). For qPCR, the PowerUp SYBR Green Master Mix (Applied Biosystems; Thermo Fisher Scientific, Inc.) was used to measure ERP72,
ICAM1, VCAM1, and SELE gene expression levels 15, 30, 90 and $120 \mathrm{~min}$ post-injury. Fluorescence was monitored for 40 cycles $\left(95^{\circ} \mathrm{C}\right.$ for $3 \mathrm{sec}, 60^{\circ} \mathrm{C}$ for $\left.30 \mathrm{sec}\right)$ on a 7500 fast real-time PCR system (Applied Biosystems; Thermo Fisher Scientific, Inc.). Experiments were run in triplicates for all groups. The data were quantified using the $2^{-\Delta \Delta \mathrm{Cq}}$ method (40), and the results are presented as the fold change relative to GAPDH, using the untreated control group as reference.

Western blotting. Total proteins were extracted from homogenized vessels using TRIzol as recommended by the manufacturer (Invitrogen; Thermo Fisher Scientific, Inc.). Protein extracts from two rats of each group were resuspended in $1 \%$ SDS supplemented with phosphatase and a protease inhibitor cocktail (New England Biolabs, Inc.). The soluble protein concentration was determined using a BCA Protein Assay kit (Thermo Fisher Scientific, Inc.). For western blotting analysis, $20 \mu \mathrm{g}$ of total protein extract were resolved by SDS-PAGE on $12 \%$ gels (41). The proteins were transferred to nitrocellulose membranes, blocked for $1 \mathrm{~h}$ at room temperature in PBS containing 5\% non-fat dry milk and 0.1\% Tween-20, then incubated overnight at $4^{\circ} \mathrm{C}$ with the anti-ERp72 rabbit polyclonal antibody (1:1,000; Abcam; cat. no. ab82587) or anti- $\beta$-actin mouse antibody $(1: 1,000$; BD Biosciences; cat. no.612656) (41). The respective HRP-conjugated secondary antibodies (anti-rabbit IgG HRP-linked antibody cat. no. 7074 and anti-mouse IgG HRP-linked antibody cat. no. 7076; Cell Signaling Technology, Inc.) were used at a dilution of 1:1,000 for $2 \mathrm{~h}$ at room temperature for the detection step. The bands were detected using an Enhanced Chemiluminescence kit (Cytiva) and images were acquired using an LAS-1000 plus image analyzer with Image Reader LAS-1000 Lite software ver. 2.2 (Fujifilm Wako Pure Chemical Corporation).

Multiplex immunohistochemistry. Immunohistochemistry staining was performed on the Ventana Discovery Ultra Chromogenic AmpHQ automated immunostainer (Ventana Medical Systems, Inc.) to determine ERp72 protein expression $2 \mathrm{~h}$ post-injury in the aforementioned sections. The multiplex technology uses the sequential application of unmodified 
primary antibodies with specific heat deactivation steps in between that does not affect the epitope in the tissue (42). In a sequential staining procedure, deactivation of the primary antibody and HRP/AP-conjugated secondary antibody bound to the first biomarker, before the application of subsequent biomarker(s), is critical to reducing cross-reactivity and facilitating downstream image analysis (43). Deparaffinization and on-board antigen retrieval were performed for $64 \mathrm{~min}$ at $95^{\circ} \mathrm{C}$ using the $\mathrm{CC} 1$ reagent (Ventana Medical Systems, Inc.; cat. no. 950-500). Blocking buffer (Invitrogen; Thermo Fisher Scientific, Inc.; cat. no. 00-4952-54) was used for section blocking at $37^{\circ} \mathrm{C}$ for $20 \mathrm{~min}$. Slides were processed using Ventana Medical Systems reagents except as noted, according to the manufacturer's instructions. The Cell Conditioning (CC) 2 buffer (Ventana Medical Systems, Inc.; cat. no. 950-123) was used for deactivation of the bound primary antibody and secondary antibody-HRP while maintaining the integrity of the tissue morphology and the subsequent epitopes (42).

The pre-diluted anti-ERp72 primary antibody $(1: 10,000$; Abcam; cat. no. ab109869), was applied first at $37^{\circ} \mathrm{C}$ for $60 \mathrm{~min}$, followed by $16 \mathrm{~min}$ incubation at $37^{\circ} \mathrm{C}$ with ready-to-use OmniMap anti-Rabbit HRP solution (Ventana Medical Systems, Inc.; cat. no. 760-4311) followed by $8 \mathrm{~min}$ with ready-to-use ChromoMap DAB kit (Ventana Medical Systems, Inc.; cat. no. 760-159) for single IHC or DISCOVERY Teal HRP Kit (RUO; Ventana Medical Systems, Inc.; cat. no. 760-247) for duplex IHC (42). For duplex IHC, a supplementary treatment with mouse anti-CD34 (1:400; Abcam; cat. no. ab8536) for $40 \mathrm{~min}$ at $37^{\circ} \mathrm{C}$ was performed and followed by 16 min incubation with a secondary ready-to-use OmniMap anti-Ms HRP antibody (Ventana Medical Systems, Inc.; cat. no. 760-4310) and 8 min with ready-to-use ChromoMap DAB kit (Ventana Medical Systems, Inc.; cat. no. 760-159). Finally, the slides were counterstained with hematoxylin and bluing reagent according to the manufacturer's recommendations. Images of IHC specimens were captured on an Olympus BX-51 microscope (Olympus Corporation) fitted with a CoolSNAP ES2 CCD camera with 1,392x1,040 pixels and 12-bit resolution (Teledyne Photometrics) and Olympus UPlanSApo 20x (NA 0.75) and 10x (NA 0.40) air objectives (Olympus Corporation). Image acquisition and quantitative analysis were performed using $\mathrm{HALO}^{\mathrm{TM}}$ Image Analysis software Cytonuclear module v. 1.6 (Indica Labs). Blood vessels were manually delimited on the acquired images and endothelial cells were identified by their morphology and counted, then classified according to the level of expression of ERp72 into low (1+), medium (2+) and high expression cells (3+) by quantifying color intensity of each counted cell.

Multiplex immunofluorescence. For immunofluorescence, deparaffinization and on-board antigen retrieval were carried out for $64 \mathrm{~min}$ at $95^{\circ} \mathrm{C}$ using the $\mathrm{CC} 1$ reagent (Ventana Medical Systems, Inc.; cat. no. 950-500). Blocking buffer (Invitrogen; Thermo Fisher Scientific, Inc.; cat. no. 00-4952-54) was used for section blocking at $37^{\circ} \mathrm{C}$ for $20 \mathrm{~min}$. Slides were processed using Ventana Medical Systems reagents according to the manufacturer's instructions. A duplex protocol was used; two pre-diluted primary antibodies were sequentially applied for $40 \mathrm{~min}$ at $37^{\circ} \mathrm{C}$ each, in the following order: Rabbit anti-ERp72 (1:10,000, Abcam; cat. no. ab109869) followed by mouse anti-CD34 (1:400; Abcam; cat. no. ab8536) for $40 \mathrm{~min}$ at $37^{\circ} \mathrm{C}$. Secondary antibodies were then added in the following order using the indicated chromogenic detection: OmniMap anti-Rb HRP (Ventana Discovery; cat. no. 760-4310) and Discovery Rhodamine kit (Ventana Medical Systems, Inc.; cat. no. 760-233), then ready-to-use OmniMap anti-Ms HRP (Ventana Medical Systems, Inc.; cat. no. 760-4310) and Discovery Cy5 kit (Ventana Medical Systems, Inc.; cat. no. 60-238). Sections were then counterstained with DAPI and mounted using Vectashield mounting medium (Vector Laboratories, Inc.). Images were acquired on a Zeiss Axio Observer Z1 Inverted Fluorescence Microscope (Carl Zeiss AG). Image acquisition was performed using HALO $^{\text {тм }}$ Image Analysis software v. 2.1 (PerkinElmer, Inc.).

Plating and maintenance of HUVECs. HUVECs were grown in EGM-2 medium (Lonza Group Ltd.). When cells reached $80-90 \%$ confluence, they were trypsinized and resuspended at $120,000 \mathrm{cells} / \mathrm{ml}$. Then, 36,000 cells/well $(0.3 \mathrm{ml})$ were plated into a 48 -well polystyrene tissue culture plate. For the treatment, HUVEC monolayers were washed once with Hank's balanced salt solution (HBSS) and supplemented with $0.3 \mathrm{ml}$ DMEM media containing $100 \mathrm{ng} / \mathrm{ml}$ TNF $\alpha$ (R\&D Systems, Inc.; cat. no. 210-TA-100) for $3 \mathrm{~h}$ before neutrophils addition. Negative controls were supplemented with DMEM alone.

Neutrophil isolation and labeling. Experimental protocols were approved by the Research and Ethics committee at Arabian Gulf University. All methods were carried out in accordance with the committee's relevant guidelines and regulations. Participants provided written informed consent prior to enrolment in the study and for the publication of the data. Isolation of neutrophils was performed using Polymorphprep ${ }^{\mathrm{TM}}$ density gradient solution (Progen Biotechnik $\mathrm{GmbH}$ ) to isolate polymorphonuclear granulocytes from whole blood (44). Briefly, $30 \mathrm{ml}$ whole blood from a healthy human volunteer were collected in EDTA and $5 \mathrm{ml}$ of whole blood were layered over $5 \mathrm{ml}$ Polymorphprep ${ }^{\mathrm{TM}}$ solution and centrifuged at $450 \mathrm{xg}$ for $30 \mathrm{~min}$ at $18-22^{\circ} \mathrm{C}$. The plasma and upper leukocyte phase containing peripheral blood mononuclear cells were removed and the lower leukocyte layer containing neutrophils was recovered. Cells were washed in PBS (without $\mathrm{Ca}^{2+}$ and $\mathrm{Mg}^{2+}$ ) and centrifuged at $450 \times \mathrm{g}$ for $10 \mathrm{~min}$ at $18-22^{\circ} \mathrm{C}$. After red blood cell lysis, cells were washed in $1 \mathrm{X}$ PBS (without $\mathrm{Ca}^{2+}$ and $\mathrm{Mg}^{2+}$ ), then centrifuged at $250 \mathrm{x}$ g for $5 \mathrm{~min}$ at $18-22^{\circ} \mathrm{C}$. After a final wash in PBS (without $\mathrm{Ca}^{2+}$ and $\mathrm{Mg}^{2+}$ ), cells were resuspended at $2 \times 10^{6}$ cells $/ \mathrm{ml}$ in RPMI-1640 (Sigma-Aldrich; Merck KGaA).

2',7'-Bis-(2-carboxyethyl)-5(and-6)-carboxyfluorescein, acetoxymethyl (BCECF-AM; Invitrogen; Thermo Fisher Scientific, Inc.) was used for neutrophil labeling. Briefly, BCECF-AM was added to the cells at a final concentration of $1 \mu \mathrm{M}$ and incubated for $30 \mathrm{~min}$ at $37^{\circ} \mathrm{C}$. Cells were washed twice in HBSS/BSA and resuspended in serum-free RPMI-1640 medium (Sigma-Aldrich; Merck KGaA) to achieve a final concentration of $1 \times 10^{6}$ cells $/ \mathrm{ml}$.

Cell adhesion assay. Adherence of BCECF-AM-labeled neutrophils to HUVEC monolayers was evaluated as described previously (45). Confluent HUVECs were prepared 
Figure 1. Yeast two-hybrid screening results. ERp72 (PDIA4) was identified as a binding partner of SI/0202. Interacting domain mapping identified the ERp72 domain ranging from aa172 to aa 352 as the SID (orange). Conserved domains are indicated in green. The transmembrane domain is indicated in red. The signal peptide is indicated in yellow. The coiled coil domain is indicated in purple. Aa, amino acid; ERp72, endoplasmic reticulum resident protein 72; PDIA4, protein disulfide isomerase family A member 4; SID, selected interacting domain.

as aforementioned and incubated at $37^{\circ} \mathrm{C} 24 \mathrm{~h}$ before use. Rabbit polyclonal antibodies specific for ERp72 (Abcam; cat. no. ab109869) were added to HUVECs in serum-free RPMI-1640 medium at different concentrations $(5-100 \mu \mathrm{g} / \mathrm{ml})$ and incubated for $20 \mathrm{~min}$ at $37^{\circ} \mathrm{C}$. Rabbit anti-ERp5 polyclonal antibody (Abcam; cat. no. ab11432) was used as an isotype control at $100 \mu \mathrm{g} / \mathrm{ml}$. HUVEC monolayers ( $\geq 80 \%$ confluence) were then washed twice with warm serum-free RPMI-1640, then added to BCECF-AM-labeled neutrophils $\left(1.0 \times 10^{5}\right.$ neutrophils/well in $100-\mu 1$ total volume). After a 15-min incubation, non-adherent neutrophils were removed using a gentle wash with PBS. After washing, $100 \mu$ l PBS was added to each well and fluorescence was determined using a fluorescence plate-reader (excitation filter was a $20-\mathrm{nm}$ bandwidth filter centered at $485 \mathrm{~nm}$, and the emission filter was a $25-\mathrm{nm}$ bandwidth filter centered at $530 \mathrm{~nm}$ ). All experiments were performed in triplicate. Wells with HUVECs that were not incubated with neutrophils were used as controls in order to obtain a background fluorescence reading. Neutrophil adhesion percentages were calculated relatively to HUVECs stimulated with $\mathrm{TNF} \alpha$ (as aforementioned) as a positive control of adhesion.

Statistical analysis. Gene expression analyses were set up in triplicates for each rat. Cell adhesion assay were run in three independent experiments. One-way ANOVA followed by Tukey's post hoc test was used to compare experimental groups to the control for all experiments. All analyses were performed using SPSS statistics software version 27 (IBM Corp.). $\mathrm{P}<0.05$ was considered to indicate a statistically significant difference.

\section{Results}

Yeast two-hybrid screening. Yeast two-hybrid screening was performed using the coding sequence of SI/0220 as a bait to screen a randomly primed HUVEC cDNA library. The screen identified ERp72 (GenBank ID 157427676) as a prey with a Predicted Biological Score (PBS) of B (high confidence in the interaction). The interacting domain mapping identified the ERp72 domain ranging from amino acids (aa) 172 to 352 as the selected interacting domain (SID) shared by all fragments matching the same reference protein (Fig. 1). This SID covers the ' $a$ ' catalytic domain and part of the ' $b$ ' substrate-binding domain.

Gene expression analysis. RT-qPCR was performed to determine the gene expression levels of ERP72, ICAM1, VCAM1

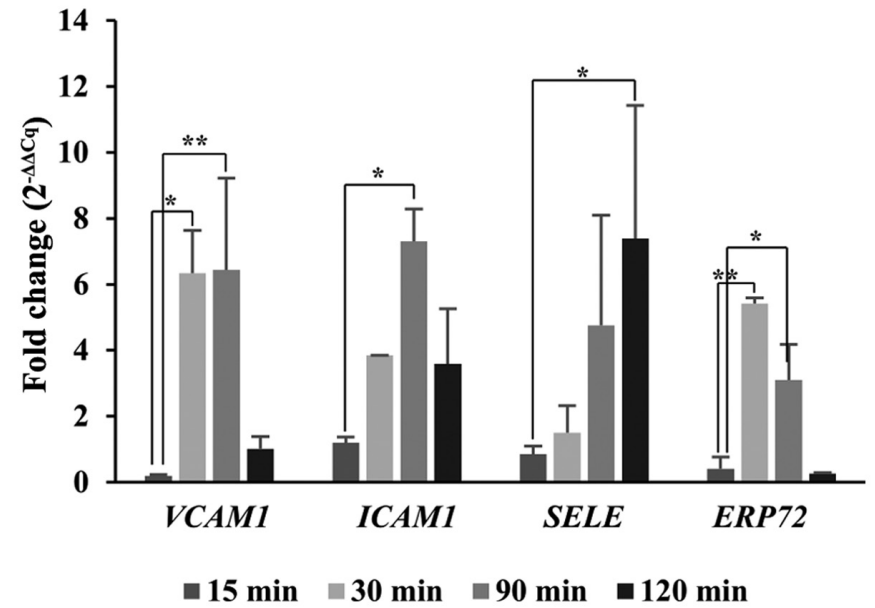

Figure 2. Gene expression profiling. ICAM1, VCAM, SELE and ERP72 gene expression levels were monitored in rat vessels in the site of injury at 15,30 , 90 and 120 min post-injury using the untreated control group as reference (not shown). Results are expressed in fold change compared to the control group and using GAPDH as a reference gene. ${ }^{*} \mathrm{P}<0.05 ;{ }^{* *} \mathrm{P}<0.01$. ERP72, endoplasmic reticulum resident protein $72 ; I C A M 1$, intercellular adhesion molecule 1; SELE, E-selectin; VCAM1, vascular cell adhesion molecule 1.

and SELE in rat blood vessels at the site of injury at different timepoints post-injury: 15, 30, 90 and $120 \mathrm{~min}$ (Fig. 2). VCAM1, ICAMI and SELE were selected to monitor the inflammation process (46). VCAMI and ICAMI expression was upregulated starting from 30 min until 90 min post-injury and reaching up to an 8-fold increase compared to the non-injured group. The SELE expression profile showed a small delay in expression, compared with VCAMI and ICAMI. Indeed, SELE expression reached a 7-fold increase at $120 \mathrm{~min}$. For ERP72, a significant upregulation was observed at $30 \mathrm{~min}$ (6-fold) and at $90 \mathrm{~min}$ post-injury (4-fold).

Multiplex immunohistochemistry and immunofluorescence. For in situ investigation, H\&E staining of muscle sections from model rat injury sites and controls was performed (Fig. 3A and B, respectively). Congestion in blood vessels was observed at the site of injury, together with infiltration of inflammatory cells in the site $2 \mathrm{~h}$ post-injury, as reported previously (35). Single immunohistochemical labeling of ERp72 indicated detectable expression of the protein mainly in the endothelial cells of the blood vessels in control sample tissues (Fig. 3D) and post-trauma tissues (Fig. 3C). CD34 (a specific marker of microvascular endothelial cells) was co-expressed with ERp72 in the endothelial cells of the blood vessels in the control group (Fig. 4A). Indeed, multiplex immunofluorescence 

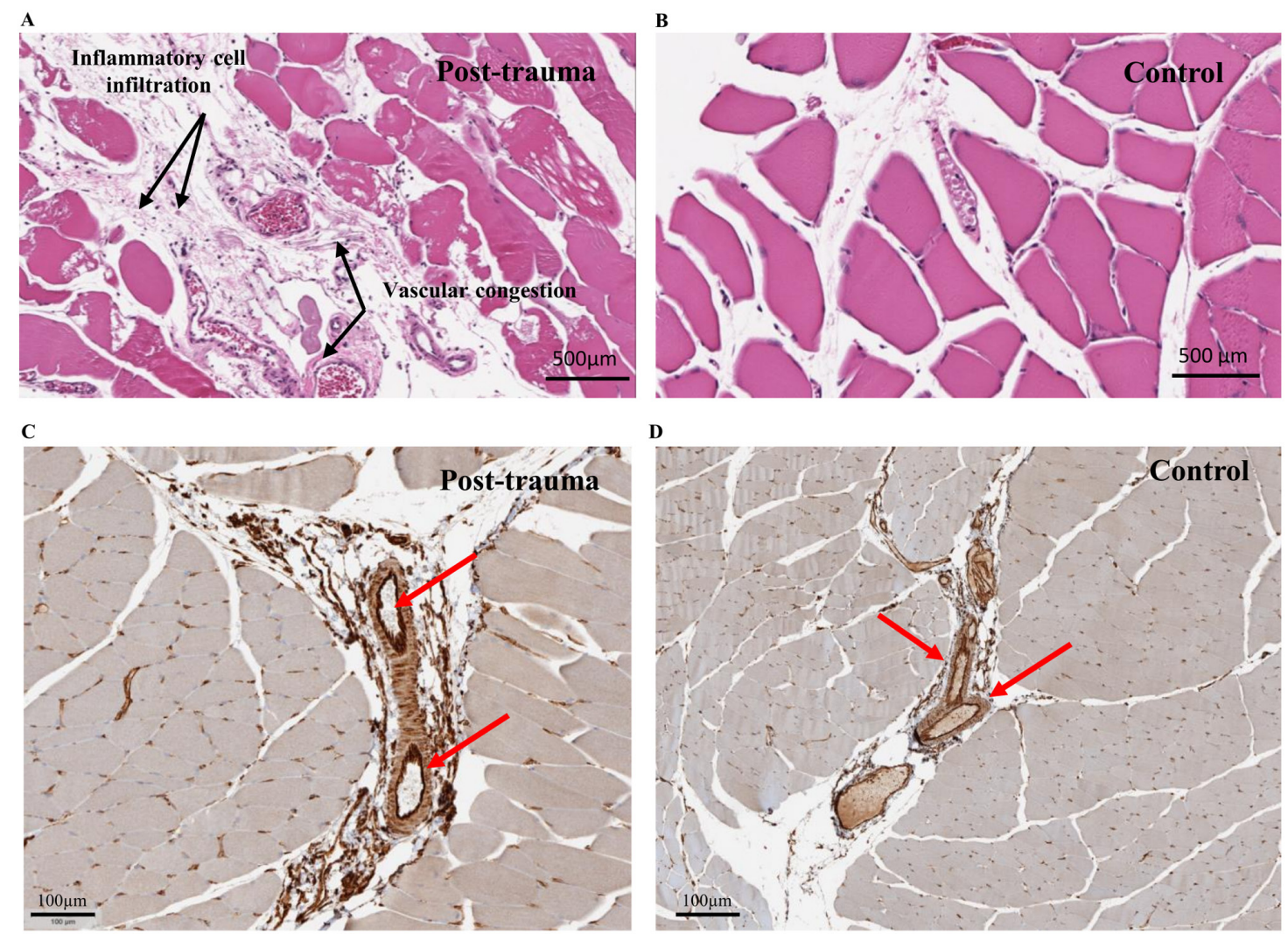

Figure 3. In situ H\&E staining of the muscle sections. Congestion was observed in blood vessels neighboring the site of injury, together with infiltration of inflammatory cells at the site (A) $2 \mathrm{~h}$ post-trauma compared with (B) the control group, as indicated by the arrows. Single immunohistochemical labeling of ERp72 protein expression is detectable (brown) in the blood vessel walls in (C) $2 \mathrm{~h}$ post-injury and (D) control samples, as indicated by the red arrows. ERp72, endoplasmic reticulum resident protein 72 .

confirmed this colocalization at the level of endothelial cells, as well as the increase of specific ERp72 signal $2 \mathrm{~h}$ post-injury (Fig. 4C) compared with the control group (Fig. 4B).

To measure differential expression of these target proteins, quantification of their cellular expression in vessels at the site of injury was performed (Fig. 5A and B). A notable increase in the percentage of cells with medium ERp72 expression (33-66\%) was observed $2 \mathrm{~h}$ post-injury. Western blot analysis also indicated ERp72 upregulation in total vessel extracts at $2 \mathrm{~h}$ post-injury compared to untreated rats (Figs. 5C, S1 and S2).

Adhesion assay. Adhesion of human neutrophils to $\mathrm{TNF} \alpha$-activated HUVECs was used to examine the role of ERp72 in cellular adhesion (Fig. 6A). Anti-ERp72 antibodytreated HUVECs that did not receive TNF $\alpha$ treatment displayed relatively low neutrophil adhesion levels (6-14\%). There was little background signal in control cells without addition of neutrophils (media only, NPH-). Interestingly, anti-ERp72 antibody significantly inhibited neutrophil adhesion starting from $10 \mu \mathrm{g} / \mathrm{ml}(\mathrm{P}<0.001)$. The highest adhesion inhibition was observed for antibody concentration of $25 \mu \mathrm{g} / \mathrm{ml}$ (35\% inhibition of neutrophil adhesion). However, no inhibition was observed for the anti-ERp5 isotype control (anti-ERp5 $100 \mu \mathrm{g} / \mathrm{ml} / \mathrm{TNF}+$ ) for the tested concentration. Light (Fig. 6B) and fluorescence microscopy (Fig. 6C-E) of neutrophils stained with BCECF-AM-labeled dye confirm the results obtained with fluorescence measurement.

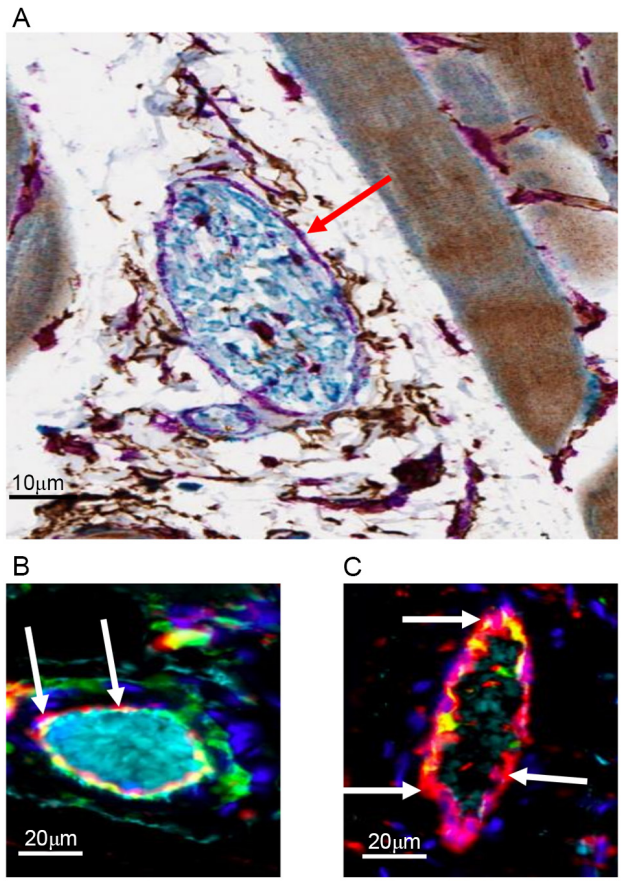

Figure 4. Multiplex immunohistochemistry and immunofluorescence. (A) Multiplex immunohistochemistry showing co-localization of ERp72 (teal) and CD34 (brown) in the endothelial cells of the blood vessels in the control tissue sample (red arrow). (B and C) Multiplex immunofluorescence using anti-ERp72 (red) and anti-CD34 (cyan) with DAPI counterstaining (blue) showing the co-localization of the two markers in the endothelial cells of the blood vessels (white arrows) (B) in control tissue before injury and (C) in tissue $2 \mathrm{~h}$ post-injury. ERp72, endoplasmic reticulum resident protein 72 . 
A

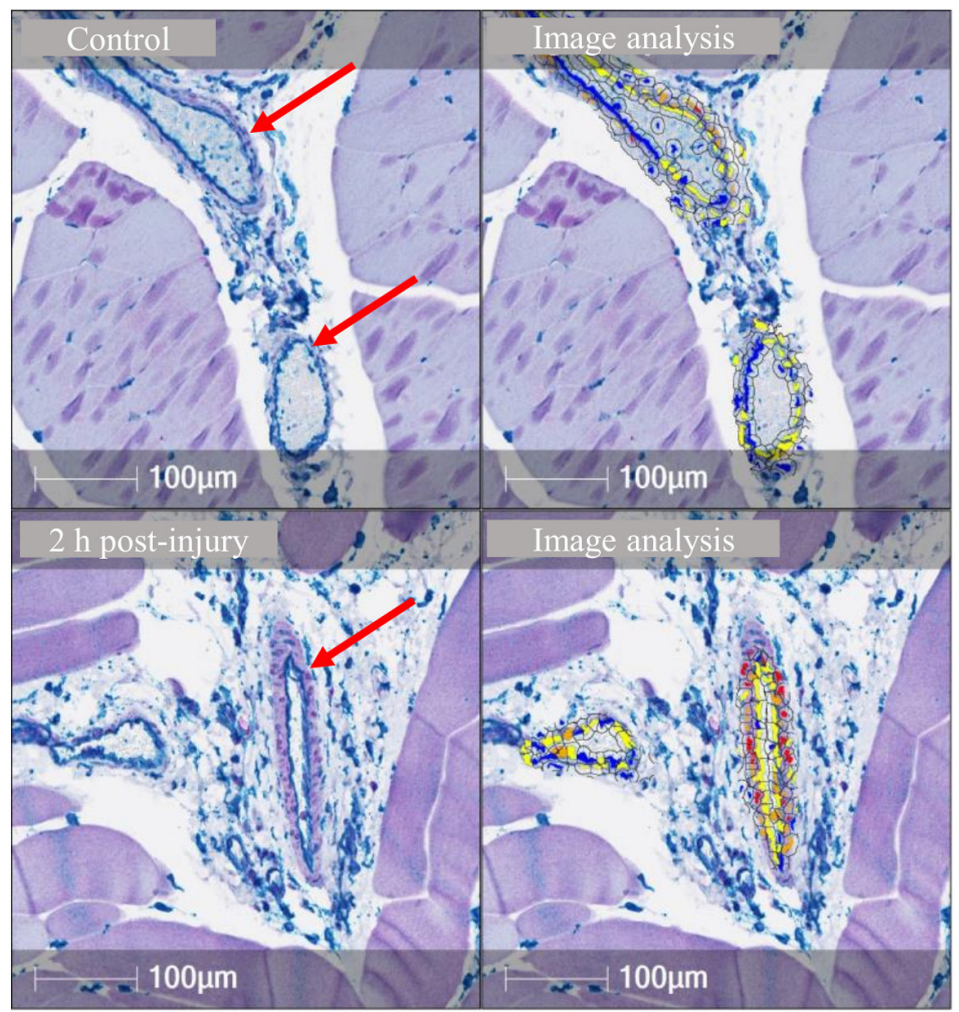

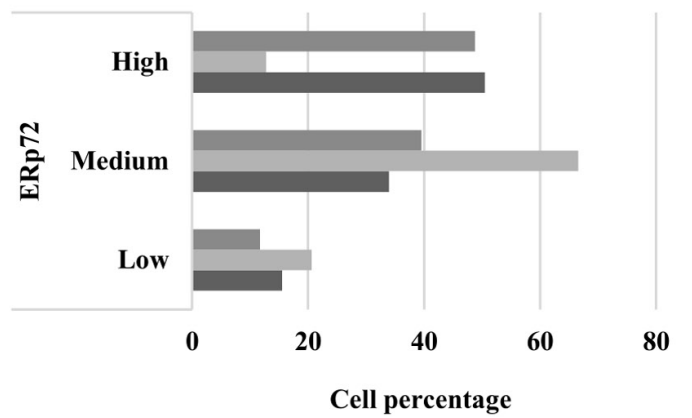

$\boxplus 4 \mathrm{~h}$ post-injury $\approx 2 \mathrm{~h}$ post-injury $\quad$ Control

C

$\begin{array}{lll}1 & 2 & 3\end{array}$

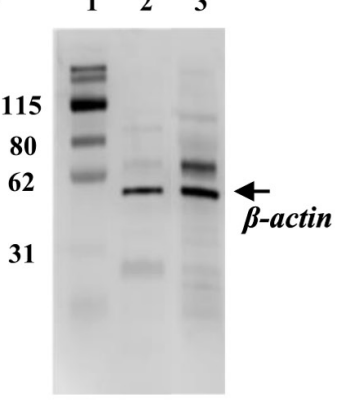

Figure 5. ERp72 expression in blood vessels. (A) Immunohistochemical quantification of ERp72 (teal) expression in endothelial cells of vessel-containing zones (red arrows) in control samples and $2 \mathrm{~h}$ post-injury. For image analysis, the levels of expression of target proteins on endothelial cells are classified as low (blue), medium (yellow) and high (red). (B) Percentage distribution of cells with high, medium and low expression of ERp72 in control, or 2 and $4 \mathrm{~h}$ post-injury. A single rat was used for the analysis from each group. (C) Western blot detection of ERp72 (right panel) and $\beta$-actin (left panel) expression in total vessels extracts $2 \mathrm{~h}$ post-injury. Lane 1, molecular weight marker; lane 2, control rat; lane 3, treated rat $2 \mathrm{~h}$ post-injury. ERp72, endoplasmic reticulum resident protein 72 .

\section{Discussion}

The development and progression of leukocyte-mediated tissue injury in inflammatory diseases is a multi-step processes that involves several adhesion molecules and neutrophil extravasation through the endothelial lining $(47,48)$. The present study was based upon an observation made during the development of a biological anti-inflammatory compound, SI/0220. This compound has the capacity to modulate PMN transmigration and to downregulate the expression of proinflammatory soluble mediators in ex vivo (data not shown; patent pending). SI/0220 was used as a prey in a yeast two-hybrid system to screen a library of HUVECs for a binding partner. This screening unexpectedly revealed ERp72 as a major binding partner of SI/0220, with a high-confidence interaction. Erp72 comprises 645 aa and is one of the largest PDI family members. The protein structural organization contains three classical Cys-Gly-His-Cys active sites, where the a- and a'-type domains are separated by the non-catalytic b-type domain (14). Unlike other PDI family members, ERp72 possesses a C-terminal Lys-Glu-Glu-Leu ER retention sequence. The protein associates with ER heat shock protein 70 (also known as binding immunoglobulin protein), Grp94, PDI and ERp29 to form a multiprotein chaperone complex that can bind to unfolded protein substrates (49). This finding suggested endothelial ERp72 might be involved in the neutrophil adhesion process acting from the endothelial side, since PDI expression in leukocytes has been demonstrated to play a role in this process (9).

To investigate the potential role of vascular ERp72 in neutrophil recruitment, ERp72 gene expression was examined in a previously developed rat model of skeletal muscle injury (35). In this mechanical trauma model, the acute inflammatory response was characterized by an early wave of PMN influx (within 30 min post-injury) into the injured site and the endomysium $5-10 \mathrm{~mm}$ from the immediate site of hematoma formation, followed by a second phase $3 \mathrm{~h}$ post-trauma that lasts up to $24 \mathrm{~h}$ (35). Histological examination of inflamed muscle of rats that received the anti-CD11b-blocking monoclonal antibody OX4238 also indicated a significant decrease in the number of infiltrating PMN in this model (35). Neutrophils are amongst the first cells to arrive at the site of muscle injury (50). In the present study, a significant upregulation in ERp72 expression in capillary endothelial tissues was observed following injury, compared with control rats. ERp72 protein expression was observed in the blood vessels within the site of injury, particularly on the vascular endothelial cells. ERp72 upregulation was also confirmed using western blotting $2 \mathrm{~h}$ post-injury. Moreover, ERp72 blockade using specific antibodies inhibited neutrophil adhesion to TNF $\alpha$-activated HUVECs in a plate-based assay, similar to what has been shown with PDI (9). Thus, these data support the role of 


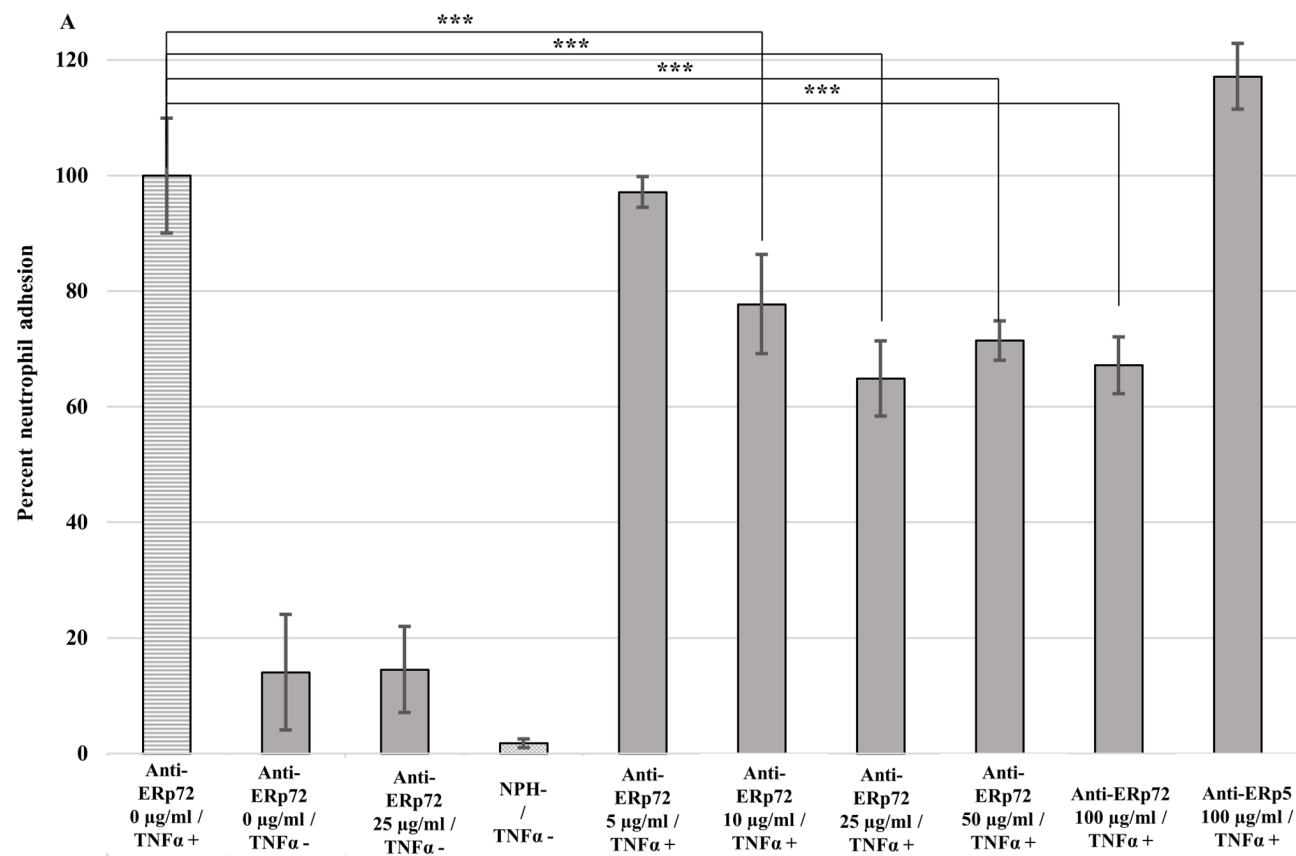

B

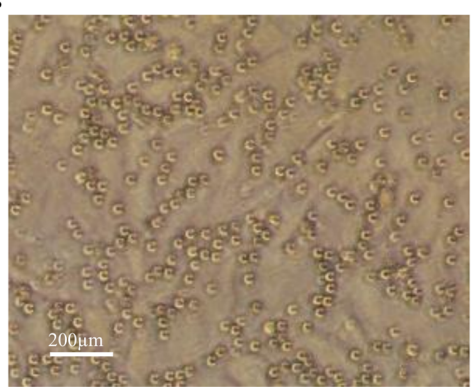

C

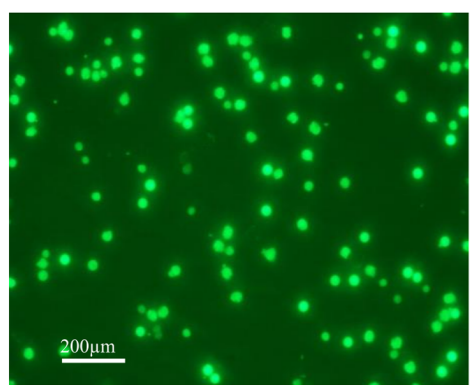

TNF $\alpha(100 \mathrm{ng} / \mathrm{ml})+$ anti-ERp72 $(25 \mu \mathrm{g} / \mathrm{ml})$

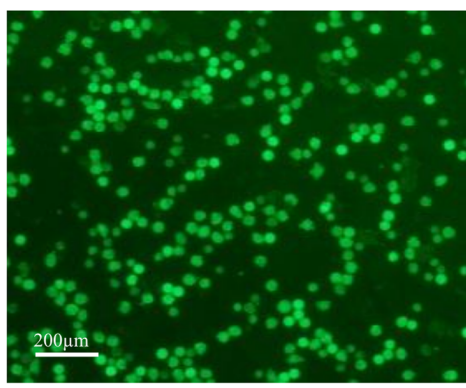

TNF $\alpha, 100 \mathrm{ng} / \mathrm{ml}$

E

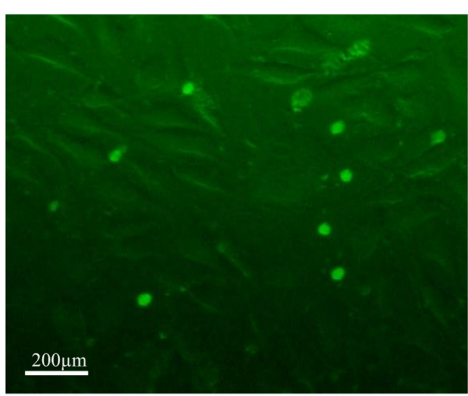

No TNF $\alpha$

Figure 6. Anti-ERp72 blocks neutrophil adhesion. (A) Neutrophil adhesion to TNFa-activated HUVECs was quantified in the presence of different concentrations of anti-ERp72 antibody. Data are presented as a percentage of neutrophil adhesion to TNF $\alpha$-activated control cells without antibody treatment (anti-ERp72 $0 \mu \mathrm{g} / \mathrm{TNF} \alpha+$ ). Negative controls (anti-ERp72 $0 \mu \mathrm{g} / \mathrm{ml} / \mathrm{TNF} \alpha+$, anti-ERp72 $25 \mu \mathrm{g} / \mathrm{ml} / \mathrm{TNF} \alpha+$ and Neutrophil (NPH)-/TNF $\alpha-$ ) and anti-ERp5 isotype control (anti-ERp5 $100 \mu \mathrm{g} / \mathrm{ml} / \mathrm{TNF} \alpha+$ ) are shown. ${ }^{* * *} \mathrm{P}<0.001$ ). (B) Representative light microscopy image of neutrophils following treatment with $100 \mathrm{ng} / \mathrm{ml} \mathrm{TNF} \alpha$. HUVECs are the elongated cells observed in the background. Representative fluorescence microscopy images of (C) neutrophils stained with BCECF-AM-labeled dye following treatment with $100 \mathrm{ng} / \mathrm{ml} \mathrm{TNF} \alpha$, (D) $100 \mathrm{ng} / \mathrm{ml} \mathrm{TNF} \alpha$ and $25 \mu \mathrm{g} / \mathrm{ml}$ anti-ERp72 antibody, or (E) without any treatment as a control. BCECF-AM, 2',7'-bis-(2-carboxyethyl)-5(and-6)-carboxyfluorescein acetoxymethyl; ERp72, endoplasmic reticulum resident protein 72; HUVEC, human umbilical vein endothelial cell.

ERp72, a thiol isomerase on the surface of endothelial cells, in neutrophil recruitment under inflammatory conditions.

The role of PDIs in inflammation and cellular adhesion has been investigated in several previous studies. Passam et al (51) demonstrated that platelet- and endothelial cell-derived ERp5 support thrombus formation in a laser-induced mouse model of thrombosis. Moreover, PDI on the surface of leukocytes regulates neutrophil recruitment during vascular inflammation through binding to $\alpha \mathrm{M} \beta 2$ (9). Moreover, the deletion of the
PDI protein in neutrophils inhibited cell adhesion to inflamed endothelium without affecting the synthesis of $\alpha \mathrm{M} \beta 2$ and other integrins, which demonstrates that PDI is essential to adhesion of human neutrophils under shear and static conditions and for binding of soluble fibrinogen to activated $\alpha \mathrm{M} \beta 2$ integrin (9). In addition, Bennett et al (52) reported that neutrophil PDIs may influence L-selectin shedding by regulating the activity of TNF $\alpha$-converting enzyme, suggesting a role of PDI in neutrophil rolling in inflammation. However, very few studies 
have highlighted the role of PDIs in endothelial cells during vascular inflammation.

Gene expression profiling demonstrated that the majority of upregulated genes in activated human coronary artery endothelial cells exposed to proinflammatory stimuli encode surface, adhesion, and receptor proteins (53). Cell adhesion molecules (CAMs) and selectins are among the most important molecules required for leukocyte adhesion, rolling and arrest (54). In the present study, upregulation of VCAM1 (30 min post-injury, ICAMI (90 min post-injury), and SELE (120 min post-injury) were observed, which validates the inflammatory state of the isolated tissues in this study. In addition, a previous study (35) reported that the adhesion process occurs in the studied model and led to the infiltration of neutrophils to the site of injury. For ERp72, upregulation of expression was observed at the same timepoints (30 and 90 min post-injury) in the same tissues and demonstrated that ERp72 protein was exclusively expressed on the endothelial cell lining of the vessels, as demonstrated by co-staining with CD34, a specific marker for endothelial cells in blood vessels (55). A significant increase in the percentage of cells with medium expression of ERp72 in blood vessels at $2 \mathrm{~h}$ post-injury. Although western blotting was conducted only $2 \mathrm{~h}$ post-injury, which constitutes a limitation to this work, it confirmed ERp72 significant expression upregulation at this time point. These results are in line with the inflammatory pattern of leukocyte infiltration in the rat model; the latter occurs in two phases: At 1 and 3 h post-injury (35). In addition, an antibody against ERp72 inhibited neutrophil adhesion to TNF $\alpha$-activated HUVECs by $35 \%$.

It has been reported that thiol exchange on integrins regulates their adhesive function $(10,56,57)$. For example, cleavage of two disulfide bonds in the cysteine-rich domain of $\alpha \operatorname{IIb} \beta 3$, induces conformational changes in the subunits and exposure of ligand-binding sites (58). PDI was demonstrated to interact with $\alpha \mathrm{M} \beta 2$ integrin in a charge-dependent manner and to regulate thiol exchange on $\alpha \mathrm{M} \beta 2$ integrin and its adhesive activity (9). Indeed, Holbrook et al and Mor-Cohen et al $(25,57)$ suggested that anti-ERp72 antibodies inhibited platelet aggregation, granule secretion, calcium mobilization, and integrin activation, revealing an important role for extracellular ERp72 in the regulation of platelet activation. Nevertheless, a similar mechanism may be proposed, whereby ERp72 similarly interacts with neutrophil integrins. ERp72 knockdown and mouse knockout experiments will help elucidate this mechanism. In addition, further characterization of ERp72 binding partners on the surface of leukocytes and identification of specific modulators for each of the PDI family members could provide further insight into the mechanisms through which this class of proteins regulates neutrophil recruitment and adhesion on the endothelial cells during vascular inflammation, and may lead to the identification of novel site-specific targets for modulation of cell-mediated chronic inflammatory diseases.

\section{Acknowledgements}

The authors thank Mr. Ammar Marweni from Arabian Gulf University (Manama, Bahrain) for his support in the work involving animals and Mrs. Luma Fayez Al Salah from Arabian Gulf University (Manama, Bahrain) for language editing.

\section{Funding}

This work was funded by an internal research grant from Arabian Gulf University (grant no. LS_NB_18).

\section{Availability of data and materials}

The datasets used and/or analyzed during the current study are available from the corresponding author on reasonable request.

\section{Authors' contributions}

NBK conceived the study and designed the experimental work and data analysis. DAM carried out experimental work and data analysis. DMF conceived the study and analyzed the data. All authors read and approved the final manuscript.

\section{Ethics approval and consent to participate}

Experimental protocols were approved by the Research and Ethics committee at Arabian Gulf University (Manama, Bahrain). All methods were carried out in accordance with the committee's relevant guidelines and regulations. Participants provided written informed consent prior to enrolment in the study and for the publication of the data.

\section{Patient consent for publication}

Participants provided written informed consent for the publication of the data.

\section{Competing interests}

The current work used information related to a biological compound cited in patent FR2909672A1 submitted by Professor Dahmani Fathallah (Department of Life Sciences, Arabian Gulf University, Bahrain), Dr M. Ali Jarboui (Institut Pasteur, Tunis, Tunisia) and Professor Koussay Dellagi (Institut Pasteur, Tunis, Tunisia) on 11/12/2006 and filed by Institut Pasteur of Tunis, which is currently undergoing renewal.

\section{References}

1. Mitroulis I, Alexaki VI, Kourtzelis I, Ziogas A, Hajishengallis G and Chavakis T: Leukocyte integrins: Role in leukocyte recruitment and as therapeutic targets in inflammatory disease. Pharmacol Ther 147: 123-135, 2015.

2. Kansas GS: Selectins and their ligands: Current concepts and controversies. Blood 88: 3259-3287, 1996.

3. Zarbock A and Ley K: Neutrophil adhesion and activation under flow. Microcirculation 16: 31-42, 2009.

4. Chiu J and Hogg PJ: Allosteric disulfides: Sophisticated molecular structures enabling flexible protein regulation. J Biol Chem 294: 2949-2960, 2019.

5. Edwards BS, Southon EA, Curry MS, Salazar F, Gale JM, Robinson MK, Graf LH Jr and Born JL: Oxidant inhibition of alphaLbeta2 integrin adhesion: Evidence for coordinate effects on conformation and cytoskeleton linkage. J Leukoc Biol 63: 190-202, 1998.

6. Schwartz BR and Harlan JM: Sulfhydryl reducing agents promote neutrophil adherence without increasing surface expression of CD11b/CD18 (Mac-1, Mo1). Biochem Biophys Res Commun 165: $51-57,1989$. 
7. Shimaoka M, Lu C, Palframan RT, von Andrian UH, McCormack A, Takagi J and Springer TA: Reversibly locking a protein fold in an active conformation with a disulfide bond: Integrin alphaL I domains with high affinity and antagonist activity in vivo. Proc Natl Acad Sci USA 98: 6009-6014, 2001.

8. Shimaoka M, Lu C, Salas A, Xiao T, Takagi J and Springer TA: Stabilizing the integrin alpha $\mathrm{M}$ inserted domain in alternative conformations with a range of engineered disulfide bonds. Proc Natl Acad Sci USA 99: 16737-16741, 2002.

9. Hahm E, Li J, Kim K, Huh S, Rogelj S and Cho J: Extracellular protein disulfide isomerase regulates ligand-binding activity of $\alpha \mathrm{M} \beta 2$ integrin and neutrophil recruitment during vascular inflammation. Blood 121: 3789-3800, S1-S15, 2013.

10. Rosenberg N, Mor-Cohen R, Sheptovitsky VH, Romanenco O, Hess $\mathrm{O}$ and Lahav $\mathrm{J}$ : Integrin-mediated cell adhesion requires extracellular disulfide exchange regulated by protein disulfide isomerase. Exp Cell Res 381: 77-85, 2019.

11. Parakh S and Atkin JD: Novel roles for protein disulphide isomerase in disease states: A double edged sword? Front Cell Dev Biol 3: 30, 2015

12. Cai H, Wang CC and Tsou CL: Chaperone-like activity of protein disulfide isomerase in the refolding of a protein with no disulfide bonds. J Biol Chem 269: 24550-24552, 1994.

13. McLaughlin SH and Bulleid NJ: Thiol-independent interaction of protein disulphide isomerase with type X collagen during intra-cellular folding and assembly. Biochem J 331: 793-800, 1998.

14. Kozlov G, Määttänen P, Thomas DY and Gehring K: A structural overview of the PDI family of proteins. FEBS J 277: 3924-3936, 2010.

15. Anelli T, Alessio M, Mezghrani A, Simmen T, Talamo F, Bachi A and Sitia R: ERp44, a novel endoplasmic reticulum folding assistant of the thioredoxin family. EMBO J 21: 835-844, 2002

16. Vaux D, Tooze J and Fuller S: Identification by anti-idiotype antibodies of an intracellular membrane protein that recognizes a mammalian endoplasmic reticulum retention signal. Nature 345 495-502, 1990

17. Cho J, Furie BC, Coughlin SR and Furie B: A critical role for extracellular protein disulfide isomerase during thrombus formation in mice. J Clin Invest 118: 1123-1131, 2008.

18. Cho J: Protein disulfide isomerase in thrombosis and vascular inflammation. J Thromb Haemost 11: 2084-2091, 2013.

19. Xiong B, Jha V, Min JK and Cho J: Protein disulfide isomerase in cardiovascular disease. Exp Mol Med 52: 390-399, 2020.

20. Zhou J, Wu Y, Wang L, Rauova L, Hayes VM, Poncz M and Essex DW: The C-terminal CGHC motif of protein disulfide isomerase supports thrombosis. J Clin Invest 125: 4391-4406, 2015.

21. Furie B and Flaumenhaft R: Thiol isomerases in thrombus formation. Circ Res 114: 1162-1173, 2014.

22. Li J, Kim K, Jeong SY, Chiu J, Xiong B, Petukhov PA, Dai X, Li X, Andrews RK, Du X, et al: Platelet protein disulfide isomerase promotes glycoprotein Ibalpha-mediated platelet-neutrophil interactions under thromboinflammatory conditions. Circulation 139: 1300-1319, 2019.

23. Kim K, Hahm E, Li J, Holbrook LM, Sasikumar P, Stanley RG, Ushio-Fukai M, Gibbins JM and Cho J: Platelet protein disulfide isomerase is required for thrombus formation but not for hemostasis in mice. Blood 122: 1052-1061, 2013.

24. Flaumenhaft R and Furie B: Vascular thiol isomerases Blood 128: 893-901, 2016.

25. Holbrook LM, Watkins NA, Simmonds AD, Jones CI, Ouwehand WH and Gibbins JM: Platelets release novel thiol isomerase enzymes which are recruited to the cell surface following activation. Br J Haematol 148: 627-637, 2010.

26. Stopa JD and Zwicker JI: The intersection of protein disulfide isomerase and cancer associated thrombosis. Thromb Res 164 (Suppl 1): S130-S135, 2018.

27. Flaumenhaft R, Furie B and Zwicker JI: Therapeutic implications of protein disulfide isomerase inhibition in thrombotic disease. Arterioscler Thromb Vasc Biol 35: 16-23, 2015

28. Wang Z, Zhang $\mathrm{H}$ and Cheng Q: PDIA4: The basic characteristics, functions and its potential connection with cancer. Biomed Pharmacother 122: 109688, 2020

29. Zhou J, Wu Y, Chen F, Wang L, Rauova L, Hayes VM, Poncz M Li H, Liu T, Liu J and Essex DW: The disulfide isomerase ERp72 supports arterial thrombosis in mice. Blood 130: 817-828, 2017.

30. Bowley SR, Fang C, Merrill-Skoloff G, Furie BC and Furie B: Protein disulfide isomerase secretion following vascular injury initiates a regulatory pathway for thrombus formation. Nat Commun 8: 14151, 2017.
31. Cho J, Kennedy DR, Lin L, Huang M, Merrill-Skoloff G, Furie BC and Furie B: Protein disulfide isomerase capture during thrombus formation in vivo depends on the presence of $\beta 3$ integrins. Blood 120: 647-655, 2012

32. Jasuja R, Furie B and Furie BC: Endothelium-derived but not platelet-derived protein disulfide isomerase is required for thrombus formation in vivo. Blood 116: 4665-4674, 2010.

33. Diamond MS, Staunton DE, de Fougerolles AR, Stacker SA, Garcia-Aguilar J, Hibbs ML and Springer TA: ICAM-1 (CD54): A counter-receptor for Mac-1 (CD11b/CD18). J Cell Biol 111: 3129-3139, 1990

34. Ivetic A, Hoskins Green HL and Hart SJ: L-selectin: A major regulator of leukocyte adhesion, migration and signaling. Front Immunol 10: 1068, 2019.

35. Zerria K, Jerbi E, Hammami S, Maaroufi A, Boubaker S, Xiong JP, Arnaout MA and Fathallah DM: Recombinant integrin CD11b A-domain blocks polymorphonuclear cells recruitment and protects against skeletal muscle inflammatory injury in the rat. Immunology 119: 431-440, 2006.

36. Vojtek AB and Hollenberg SM: Ras-Raf interaction: Two-hybrid analysis. Methods Enzymol 255: 331-342, 1995.

37. Bartel PL and Fields S: Analyzing protein-protein interactions using two-hybrid system. Methods Enzymol 254: 241-263, 1995.

38. Fromont-Racine M, Rain JC and Legrain P: Toward a functional analysis of the yeast genome through exhaustive two-hybrid screens. Nat Genet 16: 277-282, 1997.

39. Formstecher E, Aresta S, Collura V, Hamburger A, Meil A, Trehin A, Reverdy C, Betin V, Maire S, Brun C, et al: Protein interaction mapping: A Drosophila case study. Genome Res 15: 376-384, 2005.

40. Livak KJ and Schmittgen TD: Analysis of relative gene expression data using real-time quantitative PCR and the 2(-Delta Delta C(T)) method. Methods 25: 402-408, 2001

41. Liu ZQ, Mahmood T and Yang PC: Western blot: Technique, theory and trouble shooting. N Am J Med Sci 6: 160, 2014.

42. Ilié M, Beaulande M, Ben Hadj S, Chamorey E, Schiappa R, Long-Mira E, Lassalle S, Butori C, Cohen C, Leroy S, et al: Chromogenic multiplex immunohistochemistry reveals modulation of the immune microenvironment associated with survival in elderly patients with lung adenocarcinoma. Cancers 10: 326, 2018

43. Dixon AR, Bathany C, Tsuei M, White J, Barald KF and Takayama S: Recent developments in multiplexing techniques for immunohistochemistry. Expert Rev Mol Diagn 15: 1171-1186, 2015.

44. Nuzzi PA, Lokuta MA and Huttenlocher A: Analysis of neutrophil chemotaxis. Methods Mol Biol 370: 23-36, 2007.

45. Kilgore KS, Ward PA and Warren JS: Neutrophil adhesion to human endothelial cells is induced by the membrane attack complex: The roles of P-selectin and platelet activating factor. Inflammation 22: 583-598, 1998.

46. Granger DN and Senchenkova E: Inflammation and The Microcirculation. Morgan \& Claypool Life Sciences, San Rafael, CA, 2010.

47. Issekutz $\mathrm{AC}$ and Issekutz TB: The contribution of LFA-1 $(\mathrm{CD} 11 \mathrm{a} / \mathrm{CD} 18)$ and MAC-1 (CD11b/CD18) to the in vivo migration of polymorphonuclear leucocytes to inflammatory reactions in the rat. Immunology 76: 655-661, 1992.

48. Issekutz TB: Leukocyte adhesion and the anti-inflammatory effects of leukocyte integrin blockade. Agents Actions Suppl 46: 85-96, 1995.

49. Meunier L, Usherwood YK, Chung KT and Hendershot LM: A subset of chaperones and folding enzymes form multiprotein complexes in endoplasmic reticulum to bind nascent proteins. Mol Biol Cell 13: 4456-4469, 2002.

50. Londhe P and Guttridge DC: Inflammation induced loss of skeletal muscle. Bone 80: 131-142, 2015.

51. Passam FH, Lin L, Gopal S, Stopa JD, Bellido-Martin L, Huang M, Furie BC and Furie B: Both platelet- and endothelial cell-derived ERp5 support thrombus formation in a laser-induced mouse model of thrombosis. Blood 125: 2276-2285, 2015.

52. Bennett TA, Edwards BS, Sklar LA and Rogelj S: Sulfhydryl regulation of L-selectin shedding: Phenylarsine oxide promotes activation-independent L-selectin shedding from leukocytes. J Immunol 164: 4120-4129, 2000.

53. Franscini N, Bachli EB, Blau N, Leikauf MS, Schaffner A and Schoedon G: Gene expression profiling of inflamed human endothelial cells and influence of activated protein C. Circulation 110: 2903-2909, 2004.

54. Rao RM, Yang L, Garcia-Cardena G and Luscinskas FW: Endothelial-dependent mechanisms of leukocyte recruitment to the vascular wall. Circ Res 101: 234-247, 2007. 
55. Sidney LE, Branch MJ, Dunphy SE, Dua HS and Hopkinson A: Concise review: Evidence for CD34 as a common marker for diverse progenitors. Stem Cells 32: 1380-1389, 2014.

56. Mor-Cohen R, Rosenberg N, Einav Y, Zelzion E, Landau M, Mansour W, Averbukh Y and Seligsohn U: Unique disulfide bonds in epidermal growth factor (EGF) domains of $\beta 3$ affect structure and function of $\alpha \operatorname{IIb} \beta 3$ and $\alpha v \beta 3$ integrins in different manner. J Biol Chem 287: 8879-8891, 2012.

57. Mor-Cohen R, Rosenberg N, Landau M, Lahav J and Seligsohn U: Specific cysteines in beta3 are involved in disulfide bond exchange-dependent and -independent activation of alphaIIbbeta3. J Biol Chem 283: 19235-19244, 2008.
58. Yan B and Smith JW: Mechanism of integrin activation by disulfide bond reduction. Biochemistry 40: 8861-8867, 2001.

This work is licensed under a Creative Commons Attribution-NonCommercial-NoDerivatives 4.0 International (CC BY-NC-ND 4.0) License. 